\title{
Genetic Divergence in Finger Millet (Eleusine coracana (L.) Gaertn.)
}

\author{
Sneha R. Sapkal, V.V. Bhavsar* and K.K. Barhate \\ Department of Agricultural Botany, College of Agriculture, \\ Dhule-424 004 (MPKV), (M.S.), India \\ *Corresponding author
}

\section{A B S T R A C T}

An experiment was undertaken by utilizing forty finger millet genotypes for eleven yield and yield contributing characters to assess genetic divergence. The analysis of variance has shown that there was significant variation among the genotypes in all the traits. The multivariate analysis carried out using Mahalanobis D2-statistics, indicated wider genetic diversity in the genotypes of finger millet. Out of six cluster formed, cluster I was largest with twenty three genotypes, followed by cluster II with eight genotypes, cluster V with

\section{Keywords}

Genetic diversity, $\mathrm{D}^{2}$ value, Cluster, Genotype

Article Info

Accepted:

18 May 2019

Available Online:

10 June 2019 four genotypes, cluster VI with three and III, IV were mono-genotypic. The clustering pattern indicated absence of relationship between genetic diversity and geographical origin of the genotypes. The maximum inter cluster distance was observed between cluster II and VI $\left(D^{2}=26.69\right)$ while, lowest divergence was noticed between cluster I and IV $\left(D^{2}=9.43\right)$. Maximum intra cluster distance observed within cluster VI $\left(D^{2}=9.18\right)$ while lowest intra cluster distance was observed within cluster II $\left(\mathrm{D}^{2}=6.91\right)$. The variance for cluster means were high for grain iron content (41.54),followed by grain yield per plant (27.82), main ear head length (11.41), $100 \mathrm{ml}$ volume weight (9.23) and was low for number of fingers per earhead, grain calcium content, number of tillers per plant, number of productive tillers per plant. Based on inter-cluster distances, cluster mean and per se performance, and divergence class the genotypes viz., DHFM-36, DHFM-13, DHFM-18, DHFM-3, DHFM-12 were distinct and diverse and can be classified as promising genotypes. These five genotypes can be used for inter-crossing to obtain heterosis and also wider variability in moth bean. Hybridization between the genotypes of cluster VI with the genotypes of cluster III may result in exploiting more heterosis with maximum genetic divergence and are likely to produce desirable transgressive segregants in segregating generations for further crop improvement.

\section{Introduction}

Millet is a collective term referring to a number of small seeded annuals grasses that are cultivated as grain crops, primarily on marginal lands in dry areas in temperate, subtropical and tropical regions. Finger millet,
(Eleusine coracana), is also known as African millet, ragi, nachani, nagali. It is important staple food in parts of eastern and Central Africa and India. Finger millet is very adaptable to a wide range of environmental and climatic conditions, thrives at higher elevations than most other tropical cereals and 
tolerates salinity better than moist cereals. It is important cereal in Karnataka. It is intensively grown in Karnataka, Tamil Nadu, Andhra Pradesh, Orissa, Bihar, Gujarat, Maharashtra and in the hilly regions of Uttar Pradesh, Himachal Pradesh with a total area of 2.5 million hectares and 2.2 million tones of production. Finger millet (Eleusine coracana (L.) Gaertn.), is one among highly utilized belong to family Poaceae and it ranks $4^{\text {th }}$ in the importance of world. Finger millet is originated from Ethiopia. It is allopolyploid with chromosome number $2 n=4 x=36$ and evolved from a cross between two diploid species Eleusine indica (AA) and Eleusine floccifolia or Eleusine tristachya (BB) as genome contributors

(Hiremat and Salimath, 1992). Finger millet is mostly self pollinating with some amount of cross pollination (1\%) mediated by wind (Jansen and Ong, 1996, Purseglove, 1972). In India, the total area under finger millet was 1.9 million hectares with production of 1.9 million tonne and productivity $1661 \mathrm{~kg} / \mathrm{ha}$ (2017). Out of these it is cultivated in Maharashtra is 966 ha and the production is 1008 tonne and productivity under Maharashtra state was $1043 \mathrm{~kg} / \mathrm{ha}$. (20142015).

Genetic diversity which is pre-requisite for any successful breeding programme is of paramount importance. Genetic divergence among the parents play a vital role in cultivar improvement because a cross involving genetically diverse parents is likely to generate more variability in segregating generations, and also which can be used for the desired improvement. Generally, plant breeders select the parents on the basis of phenotypic diversity. Hence the knowledge of genetic diversity among the parents with respect to characters which are to be improved is essential. Keeping these things in the view, an effort has been made in the present study to evaluate a set of moth bean genotypes with the objective to study the nature and magnitude of divergence among the genotypes of finger millet.

\section{Materials and Methods}

The experimental material comprising forty genotypes of finger millet were grown in Randomized Block Design with three replications at the research farm of Department of Genetics and plant breeding, College of Agriculture, Dhule, during Kharif season of 2017. The seeds were sown by dibbling. Each entry was represented by single row of $22.5 \mathrm{~m}$ length with spacing of $10 \mathrm{~cm}$ between rows. Data were recorded on five randomly and competitive plants of each genotype from each replication for eleven quantitative characters viz., days to $50 \%$ flowering, days to maturity, plant height $(\mathrm{cm})$, number of tillers per plant, number of productive tillers per plant, main earhead length $(\mathrm{cm})$, number of fingers per earhead, $100 \mathrm{ml}$ volume weight $(\mathrm{g})$, grain yield per plant $(\mathrm{g})$, grain iron content $(\mathrm{mg} / 100 \mathrm{~g})$, grain calcium content (mg/100 g). Effective method suggested by Mahalanobis (1936) known as "Mahalanobis D2 statistics" or " $\mathrm{D}^{2}$ technique" is widely used to know genetic diversity in the germplasm. It was conducted to estimate the intra and inter cluster distances and to group the genotypes into different clusters and a logical grouping of genotypes following Tocher's method (Rao, 1952).

\section{Results and Discussion}

Analysis of variance for eleven characters indicated that the genotypes used in the present studies were significantly different (Table 1). The mean performances of 40 genotypes of finger millet for eleven characters studied are presented in Table 2. The genotype DHFM-6 (77.66 days) was the earliest for flowering (78 days) and genotype DHFM-6 (115.00 days) days to maturity (115 
days). The genotype DHFM-12 $(137.33 \mathrm{~cm})$ was found tall, genotypes DHFM-18 (8.46) exhibited significantly higher number of tillers per plant, genotype DHFM-18 (8.10) produced maximum number of productive tillers per plant, genotype DHFM-39 $(13.00 \mathrm{~cm})$ produced maximum length of main earhead, genotype DHFM-31 (10.33) recorded maximum number of fingers per earhead, genotype DHFM-27 (119.33 g) recorded maximum $100 \mathrm{ml}$ volume weight, genotype DHFM-18 (39.33 g) recorded highest grain yield, genotype DHFM-3 (12.42 $\mathrm{mg}$ ) recorded significantly high iron content, genotype DHFM-40 (416.33 mg) recorded significantly high calcium content.

On the basis of $\mathrm{D}^{2}$ values, the forty genotypes evaluated for eleven characters were grouped into six clusters by using the Tocher's method as described by Rao (1952). Cluster I was largest with 23 genotypes followed by cluster II (8 genotypes), cluster V (4 genotypes) and cluster VI (3 genotypes), while clusters III and IV were monogenotypic. In the present investigation grouping of genotypes into 6 clusters suggested the presence of substantial amount of genetic diversity in the material under investigation. The clustering pattern and the ecogeographical regions of origin of each line are given in Table 1 and 3 . Cluster I was the largest including 23 lines indicates that there was no association between clustering pattern and ecogeographical distribution of the cultures. Murty and Arunachalam (1966) and Somayajulu et al., (1970) while working with different crops, reported that geographical distribution does not necessary reflect genetic divergence. Cluster II which include 8 lines, cluster V include 4 lines and cluster VI include 3 lines under study had varieties from different eco-geographical regions, thus supporting the view that geographic distribution and genetic divergence do not follow the same trend. Wide range of diversity was reported by many workers while evaluating finger millet genotypes Naik (1991), Rao (1992), Sheriff (1992), Bandyopadhyay (1998), Vadivoo et al., (1998), Satish (2003), Sripichitt et al., (2006), Bedis et al., (2007), Sathish et al., (2007b), Kadam (2007), Anantharaju et al., (2008), Kadam (2008), Prabhu et al., (2008), Kumar et al., (2010), Sahu et al., (2012), Karad et al., (2013), Suryanarayana et al., (2014), Kumari et al., (2015), Negi et al., (2017).

The maximum intra cluster distance was observed for cluster IV $\left(D^{2}=5.12\right)$ followed by cluster III $\left(D^{2}=4.90\right)$ suggesting that genotypes present in these clusters might have different genetical architecture (Table 4). However, lowest intra cluster distance was observed in cluster $\mathrm{I}\left(\mathrm{D}^{2}=3.85\right)$ indicating that genotypes present in these cluster might have genetical similarities with one another and appeared to have evolved from common gene pool. Cluster V, VI and VII showed no intra cluster distance due to its monogenotypic nature.

Maximum inter cluster distance was observed between cluster II and VI $\left(D^{2}=26.69\right)$ followed by cluster IV and VI $\left(D^{2}=25.37\right)$, cluster I and IV $\left(D^{2}=22.90\right)$, cluster II and V $\left(\mathrm{D}^{2}=17.84\right)$, cluster III and VI $\left(\mathrm{D}^{2}=17.31\right)$, cluster V and VI $\left(D^{2}=16.91\right)$, cluster II and IV $\left(\mathrm{D}^{2}=15.88\right)$ cluster II and III $\left(\mathrm{D}^{2}=14.75\right)$ indicating, wide divergence among these clusters. These also suggest that genotype present in one cluster differ entirely from those presenting other clusters. The minimum inter cluster distance was found between cluster III and IV $\left(\mathrm{D}^{2}=13.61\right)$, cluster I and V $\left(\mathrm{D}^{2}=11.66\right)$, cluster IV and $\mathrm{V}\left(\mathrm{D}^{2}=11.44\right)$, cluster I and II $\left(\mathrm{D}^{2}=11.26\right)$, cluster I and III $\left(\mathrm{D}^{2}=11.08\right)$, cluster III and $\mathrm{V}\left(\mathrm{D}^{2}=10.87\right)$ cluster I and IV $\left(\mathrm{D}^{2}=9.43\right)$. The less inter cluster distance between these clusters revealed that genetic constitution of genotypes had close proximity. 
Based on mean performance of clusters for 11 characters (Table 5). It was observed that cluster VI exhibited the highest grain calcium content and was characterized by days to maturity, $100 \mathrm{ml}$ volume weight, days to 50per cent flowering, plant height, grain yield per plant, grain iron content, main earhead length, number of fingers per earhead, number of tillers per plant, number of productive tillers per plant. All these characters appeared to have played important role in determining grain yield of these cluster. Cluster I and cluster VI showed nearly comparable grain yield. Cluster II was characterized by less days to 50 per cent flowering, days to maturity, number of tillers plant, number of productive tillers per plant, main earhead length, grain yield per plant. Cluster IV was characterized by high days to 50 per cent flowering, days to maturity, number of tillers per plant, number of productive tillers per plant, number of fingers per earhead, grain yield per plant.

On the basis of mean performance of different clusters, it was observed that cluster IV, VI, and III were performing well for most of the characteristics.
The variance of cluster mean provides information on relative importance of different characters towards grain yield. The present study revealed that grain iron content (41.54 per cent) contributed more to genetic diversity as reflected from the Table 6 which was followed by grain yield per plant (27.82), main earhead length (11.41), $100 \mathrm{ml}$ volume weight (9.23), grain calcium content (3.72), number of fingers per earhead (3.59), number of tillers per plant (1.03), number of productive tillers per plant (1.03), days to 50 per cent flowering (0.26), plant height $(0.26)$, days to maturity $(0.13)$. These results were agreement with the observations of Jayaprakash Naik (1991) and Satish (2003) for main earhead length and number of fingers per earhead, Anantharaju and Meenakshiganesan (2008) main ear length followed by total number of fingers per earhead, grain yield per plant, grain yield per plant, main earhead lenght, number of tillers per plant, number of productive tillers per plant in finger millet (Eleusine coracana (L.) Gaertn.) (Fig. 1-3).

Table.1 Analysis of variance for eleven characters in finger millet

\begin{tabular}{|c|l|c|c|c|}
\hline \multirow{2}{*}{$\begin{array}{c}\text { Sr. } \\
\text { No }\end{array}$} & \multicolumn{1}{|c|}{ Characters } & \multicolumn{3}{|c|}{ Mean sum of square } \\
\cline { 2 - 4 } & \multicolumn{1}{|c|}{ Replication } & Genotype & Error \\
\hline $\mathbf{1}$ & Days to 50\% flowering & 33.908 & $279.59^{* *}$ & 16.429 \\
\hline $\mathbf{2}$ & Days to maturity & 33.033 & $393.21 * *$ & 35.272 \\
\hline $\mathbf{3}$ & Plant height $(\mathrm{cm})$ & 41.308 & $415.080^{* *}$ & 45.445 \\
\hline $\mathbf{4}$ & No. of tillers / plant & 0.582 & $7.679 * *$ & 0.308 \\
\hline $\mathbf{5}$ & No. of productive tillers / plant & 0.410 & $6.308^{* *}$ & 0.210 \\
\hline $\mathbf{6}$ & Main earhead length $(\mathrm{cm})$ & 0.290 & $11.546^{* *}$ & 0.272 \\
\hline $\mathbf{7}$ & No. of fingers / earhead & 0.614 & $6.986^{* *}$ & 0.374 \\
\hline $\mathbf{8}$ & 100 ml volume weight $(\mathrm{gm})$ & 16.808 & $662.402^{* *}$ & 14.859 \\
\hline $\mathbf{9}$ & Grain yield / plant $(\mathrm{gm})$ & 8.258 & $210.99 * *$ & 4.053 \\
\hline $\mathbf{1 0}$ & Grain iron content $(\mathrm{mg} / 100 \mathrm{gm})$ & 0.069 & $19.561 * *$ & 0.069 \\
\hline $\mathbf{1 1}$ & Grain calcium content $(\mathrm{mg} / 100 \mathrm{gm})$ & 1210.40 & $11756.44 * *$ & 554.75 \\
\hline
\end{tabular}

*, ** Indicates significance at 5\% and $1 \%$ level, respectively. 
Table.2 Mean performance of finger millet genotype

\begin{tabular}{|c|c|c|c|c|c|c|c|c|c|c|c|c|}
\hline $\begin{array}{l}\text { Sr. } \\
\text { No }\end{array}$ & Genotypes & $\begin{array}{l}\text { Days to } \\
50 \text { per } \\
\text { cent } \\
\text { flowering }\end{array}$ & $\begin{array}{l}\text { Days to } \\
\text { maturity }\end{array}$ & $\begin{array}{c}\text { Plant } \\
\text { height } \\
(\mathbf{c m})\end{array}$ & $\begin{array}{l}\text { No. of } \\
\text { tillers / } \\
\text { plant }\end{array}$ & $\begin{array}{c}\text { No of } \\
\text { productive } \\
\text { tillers } \\
\text { /plant }\end{array}$ & $\begin{array}{l}\text { Main } \\
\text { earhead } \\
\text { length } \\
(\mathrm{cm})\end{array}$ & $\begin{array}{c}\text { No of } \\
\text { fingers / } \\
\text { earhead }\end{array}$ & $\begin{array}{c}100 \mathrm{ml} \\
\text { volume } \\
\text { weight }\end{array}$ & $\begin{array}{l}\text { Grain } \\
\text { yield / } \\
\text { plant }\end{array}$ & $\begin{array}{c}\text { Grain iron } \\
\text { content (mg / } \\
100 \text { gm) }\end{array}$ & $\begin{array}{c}\text { Grain } \\
\text { calcium } \\
\text { content }(\mathrm{mg} \\
\text { / 100gm) }\end{array}$ \\
\hline 1 & DHFM-1 & 88.33 & 125.00 & 89.00 & 5.44 & 5.40 & 7.66 & 6.33 & 66.00 & 21.66 & 3.00 & 284.00 \\
\hline 2 & DHFM-2 & 94.66 & 125.33 & 95.33 & 6.66 & 6.00 & 7.33 & 7.33 & 113.66 & 17.90 & 11.86 & 390.00 \\
\hline 3 & DHFM-3 & 85.00 & 125.33 & 88.00 & 6.00 & 5.33 & 12.33 & 7.00 & 76.00 & 28.70 & 12.42 & 383.33 \\
\hline 4 & DHFM-4 & 94.33 & 129.66 & 101.00 & 5.66 & 5.33 & 8.00 & 6.33 & 95.66 & 19.80 & 5.52 & 354.33 \\
\hline 5 & DHRM-5 & 86.66 & 126.00 & 95.00 & 6.66 & 5.33 & 10.00 & 6.66 & 61.00 & 18.50 & 4.65 & 233.33 \\
\hline 6 & DHFM-6 & 77.66 & 115.00 & 117.33 & 2.67 & 2.33 & 6.00 & 5.00 & 107.66 & 7.00 & 3.75 & 322.00 \\
\hline 7 & DHFM-7 & 82.33 & 123.66 & 116.33 & 3.66 & 3.67 & 8.33 & 4.66 & 71.33 & 9.00 & 5.24 & 343.33 \\
\hline 8 & DHFM-8 & 93.00 & 127.66 & 120.00 & 6.66 & 5.33 & 10.00 & 6.66 & 72.00 & 20.66 & 4.31 & 308.00 \\
\hline 9 & DHFM-9 & 83.00 & 127.33 & 112.66 & 3.66 & 3.00 & 6.00 & 6.33 & 57.00 & 8.33 & 3.41 & 326.00 \\
\hline 10 & DHFM-10 & 87.66 & 127.66 & 83.66 & 4.66 & 4.66 & 9.00 & 9.33 & 79.00 & 15.20 & 3.66 & 254.66 \\
\hline 11 & DHFM-11 & 91.66 & 132.00 & 85.66 & 4.00 & 3.66 & 7.00 & 6.66 & 73.33 & 21.80 & 4.32 & 205.00 \\
\hline 12 & DHFM-12 & 92.33 & 130.00 & 137.33 & 5.66 & 5.66 & 8.33 & 6.00 & 61.00 & 20.00 & 12.35 & 402.66 \\
\hline 13 & DHFM-13 & 96.33 & 125.66 & 96.00 & 6.66 & 6.33 & 9.66 & 8.33 & 72.00 & 26.00 & 6.85 & 277.00 \\
\hline 15 & DHFM-15 & 83.00 & 125.66 & 111.66 & 4.00 & 4.00 & 9.00 & 4.00 & 82.00 & 11.00 & 2.84 & 397.00 \\
\hline 16 & DHFM-16 & 83.00 & 123.33 & 106.00 & 4.00 & 3.66 & 6.00 & 5.33 & 63.00 & 6.33 & 3.45 & 402.00 \\
\hline 17 & DHFM-17 & 94.00 & 142.33 & 110.33 & 7.00 & 5.66 & 7.33 & 6.33 & 65.00 & 19.33 & 4.20 & 415.66 \\
\hline 18 & DHFM-18 & 93.00 & 140.66 & 104.66 & 8.46 & 8.10 & 10.00 & 9.06 & 67.33 & 39.33 & 4.71 & 408.00 \\
\hline 19 & DHFM-19 & 80.00 & 117.33 & 94.33 & 3.00 & 2.67 & 7.00 & 8.00 & 69.66 & 7.00 & 3.83 & 322.00 \\
\hline 20 & DHFM-20 & 79.33 & 116.66 & 92.66 & 2.00 & 2.00 & 8.00 & 6.00 & 106.00 & 7.00 & 3.68 & 326.00 \\
\hline 21 & DHFM-21 & 92.00 & 140.66 & 101.33 & 6.46 & 6.13 & 7.66 & 7.66 & 72.00 & 21.00 & 4.87 & 247.00 \\
\hline 22 & DHFM-22 & 91.00 & 134.66 & 92.00 & 5.46 & 5.13 & 7.33 & 9.00 & 72.00 & 18.00 & 3.39 & 331.00 \\
\hline 23 & DHFM-23 & 87.00 & 127.66 & 75.33 & 6.00 & 5.33 & 6.33 & 6.66 & 74.66 & 14.00 & 2.22 & 277.00 \\
\hline 24 & DHFM-24 & 92.33 & 132.00 & 104.00 & 6.00 & 5.00 & 9.00 & 6.33 & 63.33 & 18.00 & 3.61 & 292.00 \\
\hline 25 & DHFM-25 & 92.33 & 131.00 & 104.33 & 4.00 & 3.67 & 6.66 & 8.66 & 66.00 & 18.66 & 3.40 & 305.00 \\
\hline
\end{tabular}




\begin{tabular}{|c|c|c|c|c|c|c|c|c|c|c|c|c|}
\hline 26 & DHFM-26 & 97.66 & 137.33 & 101.66 & 6.66 & 6.13 & 12.26 & 7.33 & 80.00 & 23.40 & 3.32 & 259.00 \\
\hline 27 & DHFM-27 & 91.00 & 140.00 & 95.00 & 6.06 & 5.40 & 9.00 & 5.00 & 119.33 & 19.20 & 5.58 & 364.00 \\
\hline 28 & DHFM-28 & 85.00 & 125.00 & 99.00 & 3.66 & 3.66 & 6.00 & 8.40 & 80.00 & 10.00 & 1.68 & 331.00 \\
\hline 29 & DHFM-29 & 92.66 & 131.33 & 102.00 & 6.00 & 5.20 & 8.33 & 5.00 & 80.66 & 19.66 & 3.79 & 268.33 \\
\hline 31 & $\begin{array}{l}\text { DHFM-31 } \\
\text { (Phule } \\
\text { Nachani) }\end{array}$ & 109.33 & 150.66 & 104.33 & 6.00 & 5.33 & 8.67 & 10.33 & 82.00 & 32.40 & 5.70 & 337.00 \\
\hline 32 & DHFM-32 & 113.00 & 153.33 & 88.00 & 5.00 & 5.00 & 10.33 & 7.00 & 96.00 & 31.10 & 1.52 & 277.00 \\
\hline 33 & DHFM-33 & 103.33 & 150.00 & 118.00 & 7.00 & 6.50 & 12.00 & 7.00 & 74.00 & 28.80 & 3.48 & 241.00 \\
\hline 34 & DHFM-34 & 106.00 & 148.00 & 111.66 & 6.66 & 6.33 & 11.33 & 6.33 & 70.00 & 28.20 & 4.62 & 243.00 \\
\hline 35 & DHFM-35 & 104.33 & 144.33 & 91.66 & 6.66 & 6.67 & 9.33 & 7.33 & 80.00 & 30.80 & 2.61 & 207.00 \\
\hline 36 & DHFM-36 & 112.33 & 152.33 & 106.00 & 8.00 & 7.40 & 8.40 & 10.00 & 73.33 & 33.90 & 2.46 & 295.00 \\
\hline 37 & DHFM-37 & 107.33 & 154.66 & 103.00 & 7.40 & 7.00 & 10.80 & 7.30 & 64.00 & 26.20 & 4.39 & 250.00 \\
\hline 38 & DHFM-38 & 110.00 & 156.66 & 100.33 & 7.50 & 7.20 & 11.00 & 7.66 & 63.33 & 27.00 & 3.40 & 290.00 \\
\hline 39 & DHFM-39 & 105.00 & 151.33 & 115.00 & 6.80 & 6.40 & 13.00 & 7.66 & 66.00 & 30.00 & 2.94 & 295.00 \\
\hline \multirow[t]{4}{*}{40} & DHFM-40 & 105.00 & 142.33 & 106.66 & 8.00 & 7.50 & 12.00 & 6.33 & 64.00 & 24.80 & 8.48 & 416.33 \\
\hline & S.E. \pm & 2.34 & 3.42 & 3.89 & 0.32 & 0.26 & 0.30 & 0.35 & 2.22 & 1.16 & 0.15 & 13.59 \\
\hline & $\begin{array}{c}\text { C.D. at } 5 \\
\%\end{array}$ & 6.58 & 9.65 & 10.95 & 0.90 & 0.74 & 0.84 & 0.99 & 6.26 & 3.27 & 0.42 & 38.28 \\
\hline & C.V. (\%) & 4.33 & 4.43 & 6.59 & 9.91 & 8.87 & 5.94 & 8.69 & 5.02 & 9.98 & 5.64 & 7.64 \\
\hline
\end{tabular}


Table.3 Grouping of forty finger millet genotypes into different clusters

\begin{tabular}{|c|c|c|c|}
\hline $\begin{array}{l}\text { Sr. } \\
\text { No. }\end{array}$ & Cluster & $\begin{array}{c}\text { No. of } \\
\text { genotypes }\end{array}$ & Name of genotypes \\
\hline 1 & I & 23 & $\begin{array}{l}\text { DHFM-34, DHFM-37, DHFM-33, DHFM-38, } \\
\text { DHFM-26, DHFM-39, DHFM-35, DHFM-8, } \\
\text { DHFM-5, DHFM-29, DHFM-21, DHFM-24, } \\
\text { DHFM-10, DHFM-22, DHFM-17, DHFM-11, } \\
\text { DHFM-4, DHFM-25, DHFM-1, DHFM-23, } \\
\text { DHFM-14, DHFM-30, DHFM-32. }\end{array}$ \\
\hline 2 & II & 8 & $\begin{array}{l}\text { DHFM-9, DHFM-16, DHFM-19, DHFM-28, } \\
\text { DHFM-15, DHFM-7, DHFM-20, DHFM-6 }\end{array}$ \\
\hline 3 & III & 1 & DHFM-27 \\
\hline 4 & IV & 1 & DHFM-36 \\
\hline 5 & V & 4 & DHFM-13, DHFM-31, DHFM-40, DHFM-18, \\
\hline 6 & VI & 3 & DHFM-3, DHFM-12, DHFM-2 \\
\hline
\end{tabular}

Table.4 Average intra and inter cluster distance $\left(\mathrm{D}^{2}\right.$ values $)$ for eleven characters in finger millet

\begin{tabular}{|c|c|c|c|c|c|c|}
\hline Clusters & I & II & III & IV & V & VI \\
\hline I & $\underline{7.68}$ & 11.26 & 11.08 & 9.43 & 11.66 & 22.90 \\
\hline II & & $\underline{6.91}$ & 14.75 & 15.88 & 17.84 & 26.69 \\
\hline III & & & $\underline{0.00}$ & 13.61 & 10.87 & 17.31 \\
\hline IV & & & & $\underline{0.00}$ & 11.44 & 25.37 \\
\hline V & & & & & $\underline{8.71}$ & 16.91 \\
\hline VI & & & & & & 9.18 \\
\hline
\end{tabular}

(Underlined figures indicate intra-cluster $\mathrm{D}^{2}$ values.) 
Table.5 Cluster means for eleven characters in seven clusters of forty finger millet genotypes

\begin{tabular}{|c|c|c|c|c|c|c|c|c|}
\hline \multirow{2}{*}{$\begin{array}{l}\text { Sr. } \\
\text { No }\end{array}$} & \multirow[t]{2}{*}{ Characters } & \multicolumn{6}{|c|}{ Cluster average } & \multirow[t]{2}{*}{ Cluster mean } \\
\hline & & I & II & III & IV & $\mathbf{V}$ & VI & \\
\hline 1 & Days to 50 per cent flowering & 95.86 & 81.71 & 91.00 & 112.33 & 100.92 & 90.67 & 95.41 \\
\hline 2 & Days to maturity & 137.19 & 121.75 & 140.00 & 152.33 & 139.83 & 126.89 & 136.33 \\
\hline 3 & Plant height $(\mathrm{cm})$ & 100.26 & 106.25 & 95.00 & 106.00 & 102.92 & 106.89 & 102.88 \\
\hline 4 & No. of tillers/plant & 5.93 & 3.29 & 6.07 & 8.00 & 7.28 & 6.11 & 6.11 \\
\hline 5 & No. of productive tillers/plant & 5.41 & 3.17 & 5.40 & 7.40 & 6.82 & 5.67 & 5.64 \\
\hline 6 & Main earhead length $(\mathrm{cm})$ & 9.10 & 7.04 & 9.00 & 8.40 & 10.08 & 9.33 & 8.82 \\
\hline 7 & No. of fingers/ earhead & 7.14 & 5.97 & 5.00 & 10.00 & 8.52 & 6.78 & 7.23 \\
\hline 8 & $100 \mathrm{ml}$ volume weight & 74.17 & 79.58 & 119.33 & 73.33 & 71.33 & 83.56 & 83.55 \\
\hline 9 & Grain yield/plant (gm) & 21.69 & 8.21 & 19.20 & 33.90 & 30.63 & 22.20 & 22.63 \\
\hline 10 & $\begin{array}{l}\text { Grain iron content }(\mathrm{mg} / 100 \\
\mathrm{gm})\end{array}$ & 3.83 & 3.49 & 5.59 & 2.46 & 6.44 & 12.21 & 5.67 \\
\hline 11 & $\begin{array}{l}\text { Grain calcium content } \\
(\mathrm{mg} / 100 \mathrm{gm})\end{array}$ & 273.03 & 346.17 & 364.00 & 295.00 & 359.58 & 392.00 & 338.29 \\
\hline
\end{tabular}


Table.6 Relative percent contribution of different characters towards total genetic divergence in finger millet

\begin{tabular}{|c|l|c|c|}
\hline $\begin{array}{c}\text { Sr. } \\
\text { No. }\end{array}$ & \multicolumn{1}{|c|}{ Characters } & $\begin{array}{c}\text { No. of times } \\
\text { ranked 1 }\end{array}$ & $\begin{array}{c}\text { Percent } \\
\text { contribution }\end{array}$ \\
\hline $\mathbf{1}$ & Days to 50 per cent flowering & 2 & 0.26 \\
\hline $\mathbf{2}$ & Days to maturity & 1 & 0.13 \\
\hline $\mathbf{3}$ & Plant height $(\mathrm{cm})$ & 2 & 0.26 \\
\hline $\mathbf{4}$ & No. of tillers/plant & 8 & 1.03 \\
\hline $\mathbf{5}$ & No. of productive tillers/plant & 8 & 1.03 \\
\hline $\mathbf{6}$ & Main earhead length $(\mathrm{cm})$ & 89 & 11.41 \\
\hline $\mathbf{7}$ & No. of fingers/ earhead & 28 & 3.59 \\
\hline $\mathbf{8}$ & 100 ml volume weight & 72 & 9.23 \\
\hline $\mathbf{9}$ & Grain yield/plant $(\mathrm{gm})$ & 217 & 27.82 \\
\hline $\mathbf{1 0}$ & Grain iron content $(\mathrm{mg} / 100 \mathrm{gm})$ & 324 & 41.54 \\
\hline $\mathbf{1 1}$ & Grain calcium content $(\mathrm{mg} / 100 \mathrm{gm})$ & 29 & 3.72 \\
\hline
\end{tabular}

Fig.1 Clustering by Tocher method

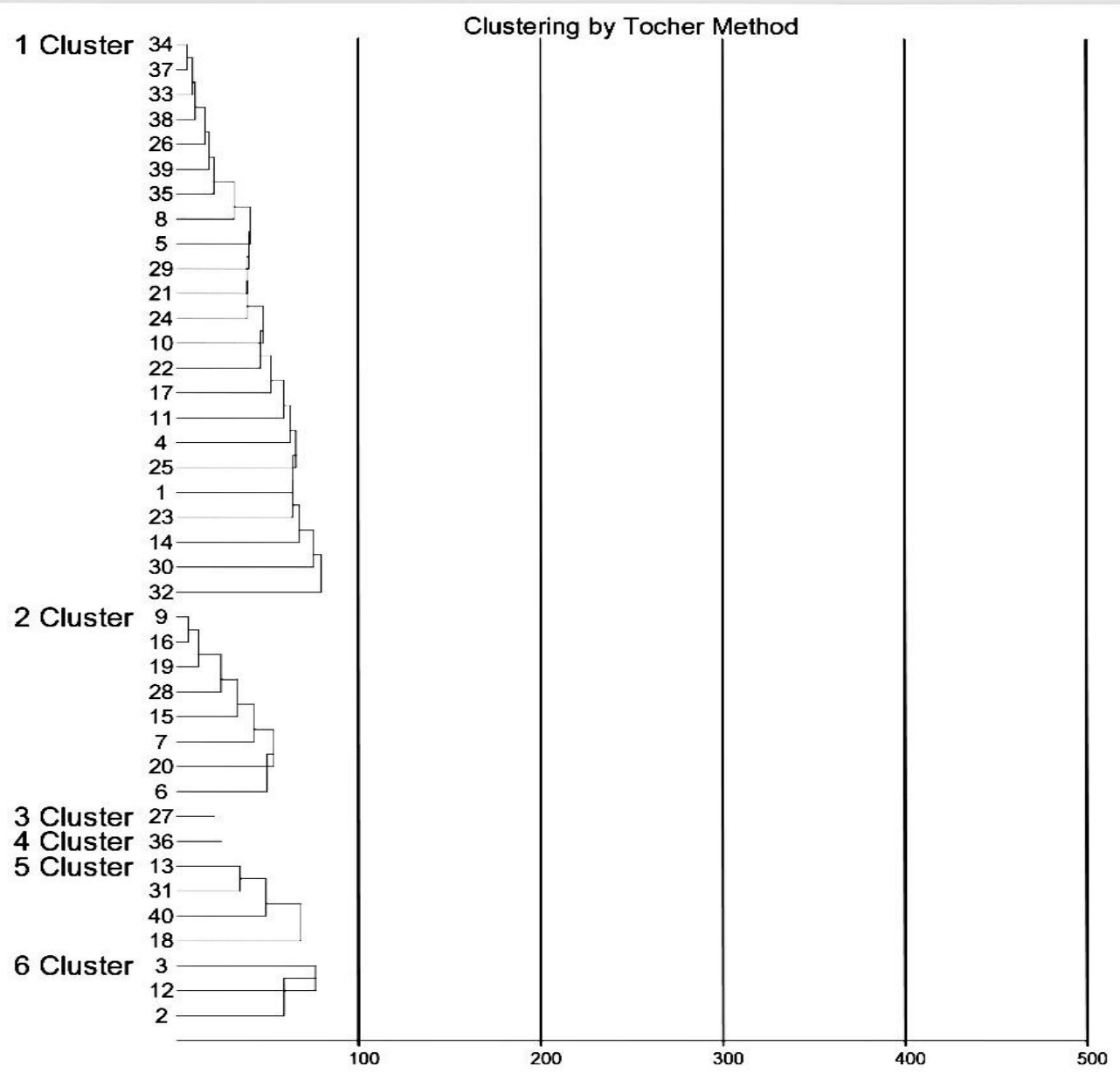


Fig.2 Divergence classes
DC 4
DC 3
DC 2
DC 1

\begin{tabular}{lcccc} 
& \multicolumn{1}{c}{} & & & \\
$\mathrm{X}$ & $\mathrm{m}-\mathrm{s}$ & $\downarrow$ & $\downarrow$ & \\
$(6.91)$ & $(8.53)$ & $\mathrm{M}$ & $\mathrm{m}+\mathrm{s}$ & $\mathrm{Y}$ \\
& & $(14.18)$ & $(19.83)$ & $(26.69)$
\end{tabular}

$\mathrm{M}=$ The mean of seven clusters and four intra-clusters (as monogenotypic cluster III and IV had no intra-cluster distance) was 14.18 .

$\mathrm{X}=$ Minimum value of distance 6.91 .

$\mathrm{Y}=$ Maximum value of distance 26.69 .

$\mathrm{S}=$ Standard deviation 5.65 .

$\mathrm{m}-\mathrm{s}=$ Mean - Standard deviation.

$\mathrm{m}+\mathrm{s}=$ Mean + Standard deviation .

Fig.3 Cluster diagram of forty germplasm in Finger millet

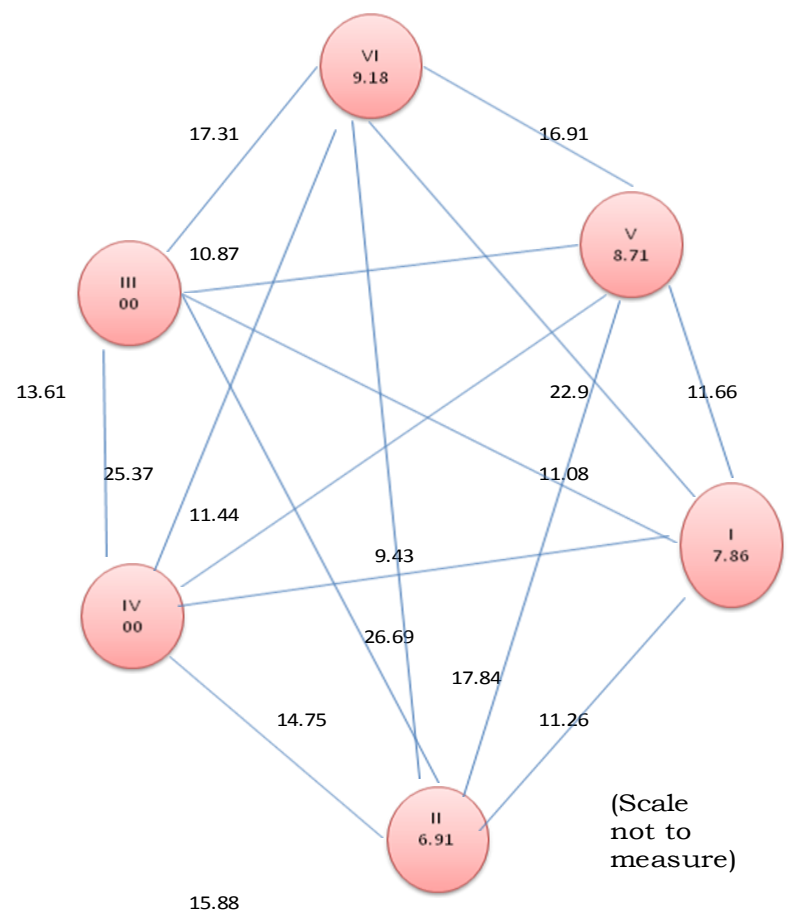


The magnitude of contribution by days to 50 per cent flowering, days to maturity, plant height was low.

The mean of six clusters and four intraclusters (as monogenotypic cluster III and VI had no intra-cluster distance) was 14.18 and standard deviation 5.65. The minimum (X) and maximum (Y) values among these distances were 6.91 and 26.69, respectively.

Grouping of cluster pairs into the divergence class (DC) is presented in Figure 2. On the light of discussion, initial choice of parents should be made from the cluster combinations falling in the divergence classes DC2 and DC3. While crossing among the genotypes of a cluster, the per se performance of the genotypes for different traits such as earliness (days to 50\% flowering and days to maturity), plant height, number of tillers per plant, number of productive tillers per plant, main earhead length, number of fingers per earhead, $100 \mathrm{ml}$ volume weight, grain yield per plant, grain iron content, grain calcium content etc. should be taken into account so, that desirable transgressive segregants would be obtained following after hybridization.

The present study revealed no parallelism between genetic divergence and geographical distribution of genotypes which was demonstrated by grouping of genotypes from same origin into different clusters separated by high genetic distance. This suggested, that genetic drift and selection in different environments may cause geographical distances.

Considering inter-cluster distances, cluster mean and per se performance, and divergence class the genotypes viz., DHMB-36, DHFM13, DHFM-18, DHFM-3, DHFM-12 were distinct and diverse and can be classified as promising genotypes. These genotypes can be used for intercrossing to obtain heterosis and also wider variability in moth bean. Hybridization between the genotypes of cluster II with the genotypes of cluster IV may result in exploiting more heterosis with maximum genetic divergence and are likely to produce desirable transgressive segregants in segregating generations for further crop improvement.

\section{References}

Allard, R. W. 1961. Relationship between genetic diversity and consistency of performance in different environments. Crop Sci. 1: 127-133

Anantharaju, P. and Meenakshiganesan, N. 2008. Genetic divergence studies in finger millet [Eleusine coracana (L.) Gaertn.]. Indian J. Agric. Res., 42(2): 120-123.

Arunachalam, V. and A. Bandopadhay. 1984. Limits to genetic divergence for occurrence of heterosis experimental evidence from crop plants. Indian $J$. Genet., 44 (3): 548-554.

Auti, S. G., Kazi, T. and Ahire, D. D. 2017. Morpho- agronomic diversity in [Eleusine coracana (L.) Gaertn. ] landraces from Maharashtra State (India). J. Sci. Agric., 1: 54-61.

Bain, A. S. and K. C. Sood. 1984. Resolution of genetic divergence for choice of parents in soybean breeding. Crop Improv., 11(1): 20-24.

Bandyopadhyay, B. B. 1998. Variability, heritability and inter relationship of important quantitative characters in finger millet. Ann. Agil. Res., 19: 173176.

Bedis, M. R., Patil, H. S., Patil, V. S. and Jangale, G. D. 2007. Genetic divergence in finger millet [Eleusine coracana (L.) Gaertn.]. Nat. J. Plant Improv., 9: 5859.

Hiremat, S.C. and Salimat S. S. 1992. The "A" genome donor of Eleusine 
coracana (L.) Gaertn. (Gramineae). Theoret. Appl. Genet. 84(5-6): 747-754.

Jansen, P. M. C. and Ong, H. C. 1996. Eleusine coracana (L.) Gaertner ev. group Finge Millet. Plant Resources of South-East Asia No 10. Cereals, Backhuys Publishers, Leiden, Netherlands. Pp.90-95.

Kadam, D. D. 2007. Genetic architecture on finger millet [Eluesine coracana (L.) Gaertn.]. Int. J. Agric. Sci., 3(2): 104107.

Kadam, D. D. 2008. Genetic divergence in finger millet (Nachani/Ragi/ Nagali/Eluesine coracana). J. Soils and Crop., 18: 138-142.

Karad, S. R. and Patil J. V. 2013. Assessement of Genetic Diversity Among Finger Millet [Eluesine coracana (L.) Gaertn.]. Genotype. Int. J. Int. Sci. Inn. Tech. Sec. C, 2(4): 37-43

Kumar, D., Tyagi, V., Ramesh, B. and Pal, S. 2010. Genetic Diversity in Finger Millet [Eleusine coracana (L.) Gaertn.]. Crop Improv., 37: 25-28 .

Kumari, S. and Singh, S. 2015. Assesment of Genetic Diversity in Promising Finger Millet [Eleusine coracana (L.) Gaertn.] Genotypes. Supplement Genet. and Plant Breeding., 10(2): 825-830.

Krishnappa, M., Ramesh, S., Chandraprakash, J. and Gowda, J., Doss, B. D. D. 2009. Genetic analysis of economic traits in finger millet. J. SAT Agril. Res., 7: 1-5.

Manimozhi, S. V., Nirmalakumari, A. and Senthil, N. 2015. Genetic Diversity for Zinc, Calcium and Iron Content of Selected Little Millet Genotypes. $J$. Nutri. Food Sci., 5:6.

Mahalanobis, P. C. 1936. On the Genaralised Distance In Science, India. 2: 49-55.

Murty, B. R. and V. Arunachalam. 1966. The nature of divergence in relation to breeding system in some crop plants. Indian J. Genet. Pl. Breed., 26: 188198.
Naik, J. B. 1991. Genetic diversity in relation to evaluation and domestication of finger millet [Eleusine coracana (L.) Gaertn.] in Africa and India. Ph. D. Thesis, Univ. Agric. Sci., Bengaluru. pp. 141.

Negi, S., Kumar, V. and Bhatt, A. 2017. Genetic Diversity among Finger Millet [Eleusine coracana (L.) Gaertn.] Genotypes for Yield and Its Contributing Traits. Int. J. Curr. Microbiol. App. Sci., 6(8): 3332-3337.

Purseglove J. W. 1972. Tropical crops: Monocotyledons. Molecular Nutrition and Food Research. 19(5-6): 395-524

Prabhu, D. A., Selvi, B. and Govindaraj, M. 2008. Genetic variability and multivariate analysis in finger millet [Eleusine coracana (L.) Gartn.]. Crop. Res., 36:1/3, 218-223.

Rao, C. R. 1952. Advanced statistical methods in Biometrical Research. John Willey and Sons. Inc., New York.

Rao, P. K. 1992. Genetic divergence in germplasm accessions of finger millet [Eleusine coracana (L.) Gaertn.]. M.Sc. (Agri.) Thesis, Univ. Agric. Sci., Bengaluru.pp. 114

Sahu, S. and Pradhan, K. 2012. Genetic divergence in finger millet [Eleusine coracana (L.) Gaertn.]. Environment and Ecology. 30(2): 291-294.

Sakamma, S., Umesh, K. B., Girish, M. R., Ravi, S. C., Satishkumar, M. and Veerabhadrappa Bellundagi. 2018. Finger Millet (Eleusine coracana L. Gaertn.) Production System: Status, Potenial, Constrainsts and Implications for I mproving Small Farmers Welfare. J. Agric. Sci., 10(1): 162-179.

Satish, D., 2003. Studies on genetic diversity based on productivity and variability for quality traits in finger millet [Eleusine coracana (L.) Gaertn.]. M.Sc. (Agri.) Thesis, University of Agricultural Science., Dharwad. pp. 129. 
Satish, D., Shanthakumar, G., Salimath, P. M., Prasad, S. G. 2007b. Genetic diversity for productivity traits in finger millet. Int. J. Plant Sci., Muzaffarnagar. 2 (2): 34-37.

Somayajulu, P. L., Joshi, A. B. and Murty, B. R. 1970. Genetic divergence in wheat. Indian J. Genet. Pl. Breed., 30: 47-48.

Sheriff, R. A. 1992. Divergence analysis in Finger millet [Eleusine coracana (L.) Gaertn.]. Indian J. Genet., 52: 72-74

Sripichitt, P. K. B., Wongyai, W. and Hongtrakul, V. 2006. Phenotypic Diversity of Ethiopian Finger Millet [Eleusine caracara (L.) Gaertn.] in Relation to Geographical Regions as an Aid to Germplasm Collection and Conservation Strategy. Kasetsart J. Nat. Sci., 41: 7-16.

Suryanarayana, L., Sekhar, D. and Venugopala Rao N. 2014. Genetic Variability and Divergence Studies in
Finger Millet [Eleusine coracana (L.) Gaertn.]. Int. J. Curr. Microbiol. App. Sci., 3(4): 931-936.

Upadhyaya, H. D., Ramesh, S., Sharma, S., Singh, S. K., Varshney, S. K., Sarma, N. D. R. K., Ravishankar, C. R., Narsimhudu, Y., Reddy, V. G., Sahrawat, K. L., Dhanalakshmi, T. N., Mgonja, M. A., Pazies, H. K., Gowda, C. C. L., and Singh, S. 2011. Genetic diversity for grain nutrients contents in core collection of finger millet [Eleusine coracana (L.) Gaertn.] germplasm. Field Crop. Res., 121: 4252.

Vadivoo, A. S., Joseph, R. and Ganesan, N. M. 1998. Genetic variability and diversity for protein and calcium contents in finger millet [Eleusine coracana (L.) Gaertn.] in relation to grain color. Plant-Foods-for-Hum. Nutrition., 52(4): 353-364

\section{How to cite this article:}

Sneha R. Sapkal, V.V. Bhavsar and Barhate, K.K. 2019. Genetic Divergence in Finger Millet (Eleusine coracana (L.) Gaertn.). Int.J.Curr.Microbiol.App.Sci. 8(06): 2230-2242.

doi: https://doi.org/10.20546/ijcmas.2019.806.266 